\title{
Production and evaluation of technological charcateristics of Fettuccine dried pasta containing bamboo fiber.
}

\section{Felipe Gianasi, Amanda Rios Ferreira, Maria Teresa Pedrosa Silva Clerici.}

\begin{abstract}
Pasta is very appreciated but due to its abundance of digestible carbohydrates, alternatives can be used to make them healthier such as the use of dietary fiber. The aim of this work was to produce and evaluate dried Fettuccine pasta, with partial replacement of semolina by white and pale yellow dietary fibers. The experiments were carried out by a physicochemical and rheological characterization of semolina and the production of dried pasta with partial replacement of fiber in $0 \%, 3.5 \%$ and $7 \%$. The samples were evaluated by their technological qualities in comparison with a standard sample made only with semolina. Statistica were made through analisys of variance (ANOVA) and Scott-Knott test $(p \leq 0.05)$. The results showed that is possible to replace the semolina in pasta for the white fibers in the analysed conditions.
\end{abstract}

\section{Key words:}

Healthy, Quality, Wheat.

\section{Introduction}

Brazil has high consumption of pasta, but, due to its glycemic index, fiber addition could make them healthier. Since pasta color is an important parameter, the aim of this work was to evaluate pasta production and characteristics with replacement of fibers.

\section{Results and Discussion}

Table 1 shows the results of pasta technological analysis. In Image 1 is possible to see images of crude and cooked pastas that presented the best technological results.

Table 1. Cooking characteristics of pasta samples* Image 1. Image of Standard sample, WFA and BFA*

\begin{tabular}{|c|c|c|c|c|c|c|c|c|c|}
\hline \multirow[t]{2}{*}{ Sample } & \multirow{2}{*}{$\begin{array}{c}\text { Optimal } \\
\text { Cooking } \\
\text { Time - } \\
\text { OCT }(\text { min) }\end{array}$} & \multirow{2}{*}{$\begin{array}{c}\text { Mass Gain - } \\
\text { MG }\end{array}$} & \multirow{2}{*}{$\begin{array}{c}\text { Volume } \\
\text { Increase - } \\
\text { VI }\end{array}$} & \multirow{2}{*}{$\begin{array}{l}\text { Loss of } \\
\text { solids } \\
\text { (g/100g) }\end{array}$} & \multirow[t]{2}{*}{ Firmness (F) } & \multicolumn{4}{|c|}{ Color Cooked Pasta } \\
\hline & & & & & & $\mathbf{L}$ & $\mathrm{a}^{*}$ & $\mathbf{b}^{*}$ & $\Delta \mathbf{E}$ \\
\hline CFA1 & 12,5 & $2,96 \pm 0,36^{\mathrm{ns}}$ & $7,25 \pm 0,34^{\text {ns }}$ & $8,81 \pm 0,60^{b}$ & $666,67 \pm 64,3^{\mathrm{b}}$ & $76,56 \pm 0,68^{c}$ & $-1,98 \pm 0,15^{\mathrm{b}}$ & $25,92 \pm 1,71^{\mathrm{b}}$ & 8,08 \\
\hline CFB1 & 12,5 & $2,96 \pm 0,01^{\mathrm{ns}}$ & $3,5 \pm 0,36^{\mathrm{ns}}$ & $5,47 \pm 0,08^{\mathrm{c}}$ & $762,6 \pm 42,59^{\mathrm{a}}$ & $75,14 \pm 0,50^{\mathrm{c}}$ & $-0,97 \pm 0,12^{\mathrm{a}}$ & $22,61 \pm 0,19^{\mathrm{c}}$ & 5,66 \\
\hline CFA2 & 13 & $2,35 \pm 0,05^{\mathrm{ns}}$ & $4,29 \pm 0,33^{\mathrm{ns}}$ & $6,07 \pm 0,53^{\mathrm{c}}$ & $654,86 \pm 24,78^{\mathrm{b}}$ & $78,65 \pm 0,99^{\mathrm{a}}$ & $-2,54 \pm 0,21^{\mathrm{c}}$ & $23,69 \pm 1,28^{\mathrm{c}}$ & 6,21 \\
\hline CFB2 & 13 & $2,91 \pm 0,01^{\mathrm{ns}}$ & $3,86 \pm 0,37^{\mathrm{ns}}$ & $11,80 \pm 2,22^{\mathrm{a}}$ & $676,72 \pm 37,9^{\mathrm{b}}$ & $76,15 \pm 2,05^{\mathrm{c}}$ & $-1,10 \pm 0,11^{\mathrm{a}}$ & $24,32 \pm 0,96^{\mathrm{c}}$ & 6,91 \\
\hline BFA1 & 13 & $2,62 \pm 0,31^{\mathrm{ns}}$ & $3,43 \pm 0,42^{\mathrm{ns}}$ & $6,70 \pm 0,09^{\mathrm{c}}$ & $567,32 \pm 41,45^{\mathrm{d}}$ & $77,90 \pm 0,64^{\mathrm{b}}$ & $-3,24 \pm 0,16^{\mathrm{d}}$ & $27,77 \pm 0,39^{\mathrm{a}}$ & 9,77 \\
\hline BFB1 & 13 & $2,82 \pm 0,29^{\mathrm{ns}}$ & $3,57 \pm 0,40^{\mathrm{ns}}$ & $7,65 \pm 0,64^{\mathrm{b}}$ & $568,86 \pm 35,54^{\mathrm{d}}$ & $80,13 \pm 0,44^{\mathrm{a}}$ & $-3,37 \pm 0,12^{d}$ & $23,28 \pm 1,01^{\mathrm{c}}$ & 6,40 \\
\hline BFA2 & 12,5 & $3,54 \pm 0,44^{\mathrm{ns}}$ & $3,71 \pm 0,38^{\mathrm{ns}}$ & $8,32 \pm 1,58^{\mathrm{b}}$ & $597,15 \pm 38,48^{\mathrm{d}}$ & $79,64 \pm 0,59^{\mathrm{a}}$ & $-3,50 \pm 0,03^{d}$ & $24,91 \pm 1,43^{\mathrm{c}}$ & 7,52 \\
\hline BFB2 & 13 & $2,85 \pm 0,09^{\mathrm{ns}}$ & $4,33 \pm 0,38^{\mathrm{ns}}$ & $7,14 \pm 0,17^{\mathrm{b}}$ & $629,02 \pm 42,78^{\mathrm{c}}$ & $79,89 \pm 1,13^{\mathrm{a}}$ & $-3,55 \pm 0,01^{d}$ & $24,45 \pm 1,92^{b}$ & 7,23 \\
\hline WFA1 & 12,5 & $2,69 \pm 0,15^{\mathrm{ns}}$ & $3,57 \pm 0,40^{\mathrm{ns}}$ & $7,35 \pm 1,23^{\mathrm{b}}$ & $664,66 \pm 52,51^{\mathrm{b}}$ & $79,11 \pm 0,19^{\mathrm{a}}$ & $-3,71 \pm 0,02^{d}$ & $24,11 \pm 1,29^{\mathrm{c}}$ & 6,56 \\
\hline WFB1 & 14 & $2,21 \pm 0,01^{\mathrm{ns}}$ & $3,14 \pm 0,45^{\mathrm{ns}}$ & $6,68 \pm 0,40^{\mathrm{c}}$ & $645,96 \pm 39,03^{\mathrm{c}}$ & $79,45 \pm 0,28^{\mathrm{a}}$ & $-3,42 \pm 0,06^{d}$ & $24,87 \pm 1,56^{\mathrm{b}}$ & 7,41 \\
\hline WFA2 & 12 & $3,03 \pm 0,37^{\mathrm{ns}}$ & $4,67 \pm 0,35^{\mathrm{ns}}$ & $5,80 \pm 0,48^{\mathrm{c}}$ & $545,44 \pm 32,05^{\mathrm{e}}$ & $81,04 \pm 0,44^{\mathrm{a}}$ & $-3,39 \pm 0,06^{\mathrm{d}}$ & $22,75 \pm 1,18^{\mathrm{c}}$ & 6,60 \\
\hline WFB2 & 12,5 & $2,89 \pm 0,07^{\text {ns }}$ & $3,38 \pm 0,37^{\mathrm{ns}}$ & $6,52 \pm 0,52^{\mathrm{c}}$ & $625,76 \pm 37,86^{\mathrm{C}}$ & $79,97 \pm 0,21^{\mathrm{a}}$ & $-2,86 \pm 026^{c}$ & $22,50 \pm 0,44^{\mathrm{c}}$ & 5,80 \\
\hline BBPA & 13 & $2,82 \pm 0,26^{\text {ns }}$ & $4,17 \pm 0,40^{\mathrm{ns}}$ & $5,65 \pm 0,19^{\mathrm{c}}$ & $603,32 \pm 57,22^{\mathrm{d}}$ & $77,88 \pm 0,51^{\mathrm{b}}$ & $-3,09 \pm 0,03^{b}$ & $24,96 \pm 1,10^{\mathrm{b}}$ & 7,05 \\
\hline ВВPB & 13 & $2,79 \pm 0,14^{\mathrm{ns}}$ & $5,2 \pm 0,38^{\text {ns }}$ & $7,88 \pm 0,88^{\mathrm{b}}$ & $673,67 \pm 44,11^{\mathrm{b}}$ & $77,20 \pm 0,10^{\mathrm{b}}$ & $-2,74 \pm 0,05^{\mathrm{c}}$ & $25,67 \pm 0,72^{\mathrm{b}}$ & 7,69 \\
\hline Standard & 11,5 & $3,13 \pm 0,20^{\mathrm{ns}}$ & $3,38 \pm 0,37^{\mathrm{ns}}$ & $6,33 \pm 0,48^{\mathrm{c}}$ & $512,64 \pm 37,3^{\mathrm{e}}$ & $76,36 \pm 0,36^{\mathrm{c}}$ & $-4,28 \pm 0,13^{d}$ & $18,18 \pm 1,18^{\mathrm{d}}$ & 0,00 \\
\hline
\end{tabular}

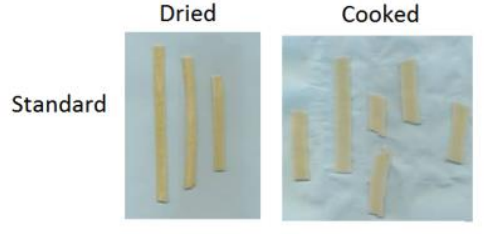

${ }^{*}$ Data expressed as mean \pm standard deviation. Different letters in the same column indicate statistical difference (Scott-Knott test, $\left.p \leq 0,05\right)$, ns=not significant. Where: CFA1 and CFB1: $80 \%$ psyllium: $20 \%$ cellulose fiber; CFA2 and CFB2: $50 \%$ psyllium: $50 \%$ cellulose fiber; BFA1 and

BFB1: bamboo fiber $60 \mu \mathrm{m}$; BFA2 and BFB2: bamboo fiber $145 \mu \mathrm{m}$; WFA1 and WFB1: wheat fiber $60 \mu \mathrm{m}$; WFA2 and WFB2: wheat fiber 145

$\mu \mathrm{m}$; BBPA and BBPB: $20 \%$ psyllium: $80 \%$ bamboo fiber. Letter A or B at the end of the sample indicates the respective concentrations: $3.5 \%$, $7.0 \%$.

Pasta with replacement of BFB and WFB presented white spots after drying. Samples with BFA and WFA in $3.5 \%$ of replacement were similar with the standard.

\section{Conclusions}

The results showed that was possible to use white and pale yellow fibers until $3,5 \%$ of replacement. Thus, BFA and WFA presented similarity with standard sample. 